\title{
Supplementary material: $\mathrm{Sr}_{3}\left[\mathrm{CO}_{4}\right] \mathrm{O}$-antiperovskite with tetrahedrally-coordinated $s p^{3}$-hybridized carbon and $\mathrm{OSr}_{6}$-octahedra
}

\author{
Dominik Spahr*,a , Jannes König ${ }^{\mathrm{a}}$, Lkhamsuren Bayarjargal ${ }^{\mathrm{a}}$, Pavel N. Gavryushkin ${ }^{\mathrm{b}, \mathrm{c}}$, Victor Milman ${ }^{\mathrm{d}}$, Hanns-Peter \\ Liermann $^{\mathrm{e}}$, Björn Winkler ${ }^{\mathrm{a}}$ \\ ${ }^{a}$ Institute of Geosciences, Goethe University Frankfurt, Altenhöferallee 1, 60438 Frankfurt, Germany \\ ${ }^{b}$ Sobolev Institute of Geology and Mineralogy, Siberian Branch of Russian Academy of Sciences, 630090 Novosibirsk, Russian Federation \\ ${ }^{c}$ Novosibirsk State University, 630090 Novosibirsk, Russian Federation \\ ${ }^{d}$ Dassault Systèmes BIOVIA, 334 Cambridge Science Park, Cambridge, United Kingdom \\ e Photon Science, DESY, Notkestrasse 85, 22607 Hamburg, Germany
}

\section{Experimental Details}

\subsection{Sample material}

We synthesized $\mathrm{SrCO}_{3}$ single crystals by hydrothermal synthesis following equation (1). ${ }^{1}$ We used analytical $\mathrm{Sr}\left(\mathrm{NO}_{3}\right)_{2}$ (99.9\% purity, Merck, Darmstadt, Germany) and $\mathrm{NH}_{4} \mathrm{HCO}_{3}$ (99.9\% purity, Merck, Darmstadt, Germany) as purchased. $\mathrm{Sr}\left(\mathrm{NO}_{3}\right)_{2}$ was dissolved in $50 \mathrm{ml}$ bidistilled water to obtain a salt solution with $0.2 \mathrm{~mol} \mathrm{l}^{-1}$ concentration and afterwards added dropwise to $100 \mathrm{ml}$ $\mathrm{NH}_{4} \mathrm{HCO}_{3}$ solution with a concentration of $0.02 \mathrm{~mol} \mathrm{l}^{-1}$ under magnetic stirring at ambient temperature.

$$
\begin{aligned}
& \mathrm{Sr}\left(\mathrm{NO}_{3}\right)_{2}+2 \mathrm{NH}_{4} \mathrm{HCO}_{3} \rightarrow \\
& \mathrm{SrCO}_{3} \downarrow+2 \mathrm{NH}_{4} \mathrm{HNO}_{3}+\mathrm{CO}_{2} \uparrow+\mathrm{H}_{2} \mathrm{O}
\end{aligned}
$$

After precipitation the resulting suspension was transferred into a $60 \mathrm{ml}$ Teflon cup which was filled up to $60 \%$ of its volume. The teflon cup was placed in a stainless steel autoclave and heated up to 493(1) K for $24 \mathrm{~h}$ before it was cooled down to ambient temperature. In a first step the autoclave was cooled down from 493(1) K to 453(1) K in $48 \mathrm{~h}$ and in a following step from $453(1) \mathrm{K}$ to ambient temperature in $24 \mathrm{~h}$. The precipitate was extracted by vacuum filtration, washed with distilled water several times and afterwards dried in an oven at 333(1) K.

The $\mathrm{SrO}$ powder (99.5\% purity, Alfa Aesar, Kandel, Germany) was analytical grade and used as purchased. The powder was directly used after opening the sealed packaging to avoid hydration of the hygroscopic powder.

\subsection{High-pressure experiments}

All high-pressure experiments were carried out using Boehler-Almax design type diamond anvil cells (DAC). ${ }^{2}$ We used DACs with 300 and $350 \mu \mathrm{m}$ culet size and rhenium as gasket material. The rhenium gaskets were preindented to a thickness of $\approx 45 \mu \mathrm{m}$ and $130 \mu \mathrm{m}$ gasket holes were drilled by a custom-built laser setup.

We placed a $\mathrm{SrCO}_{3}$ crystal with $\approx 80 \mu \mathrm{m}$ length in the center of the culet of the bottom diamond and mounted the gasket (Fig. S 1 a). Afterwards the white SrO powder was placed on top of the crystal in the gasket hole and compressed and thereby spread in the gasket hole by tightly closing the DAC. The DAC was opened again and a ruby was placed on the top diamond, near to the edge of the gasket hole for pressure determination (Fig. S 1 b). We determined the pressure by measuring the shift of the ruby fluorescence and assumed an error of $6 \%$ for non-hydrostatic conditions. ${ }^{3}$
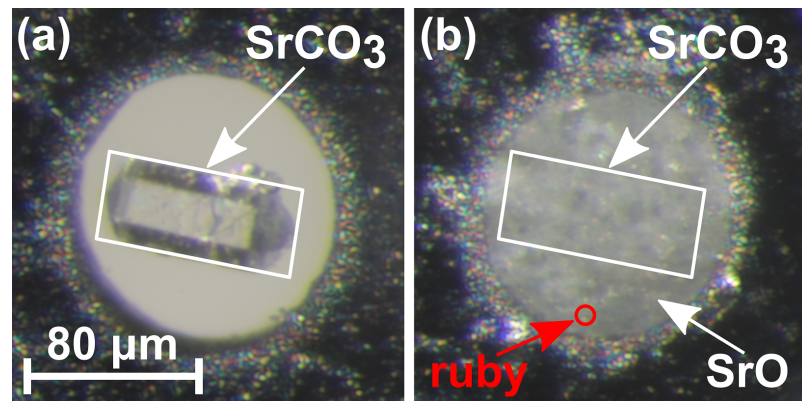

Figure S 1: (a) A transparent $\mathrm{SrCO}_{3}$ single crystal is placed in the culet's center of the bottom diamond and the gasket is mounted. (b) White SrO powder is added and compressed in the gasket hole, a ruby is added.

\subsection{Raman spectroscopy and laser heating}

For the Raman spectroscopic measurements and the double-sided laser-heating a custom built set-up was employed. ${ }^{4}$ Raman spectroscopy was carried out using an Oxxius LCX-532S Nd:YAG laser with $532.14 \mathrm{~nm}$ wavelength and a Princeton Instruments ACTON SpectraPro (SP-2356) spectrograph equipped with a Pixis 256E CCD camera. The Raman laser had a spot size of $\approx 6 \mu \mathrm{m}$ on the sample while employing a laser power of $430 \mathrm{~mW}$ (250 $\mathrm{mW}$ on the sample). Raman maps were measured using a $6 \mu \mathrm{m}$ step-size in both directions. Raman spectra were background corrected with the software Fityk. ${ }^{5}$

For double-sided laser-heating we used a Coherent Diamond $\mathrm{K}-250$ pulsed $\mathrm{CO}_{2}$ laser with $10.6 \mu \mathrm{m}$ wavelength. We applied a laser power of 1-6 W to achieve coupling of the laser to the sample in the DAC from both sides. The laser was focused on the sample to achieve a heating area of $\approx 50 \times 50 \mu^{2}$. During the laser heating we determined the highest achieved temperature $\left(T_{\max }\right)$ by the two-color pyrometer method, employing Planck and Wien fits. ${ }^{6}$

\subsection{Synchrotron X-ray diffraction}

Synchrotron powder and single crystal diffraction were performed at PETRA III (DESY) in Hamburg, Germany 
on the extreme conditions beamline P02.2. ${ }^{7}$ The beam was focused by compound reflective lenses and the resulting beam size on the sample was $7(\mathrm{H}) \times 2(\mathrm{~V}) \mu \mathrm{m}^{2}$ (FWHM). We used a Perkin Elmer XRD1621 detector and a wavelength of $0.2906 \AA$ for data acquisition and measured a $\mathrm{CeO}_{2}$ standard for calibration. The sample to detector distance was $385 \mathrm{~mm}$.

The powder samples were measured for $10 \mathrm{~s}$ while rotating them around a rotation axis perpendicular to the beam by $\pm 10^{\circ}$ to improve the counting statistics. For single crystal diffraction the samples were rotated by $\pm 35^{\circ}$. Frames were collected in $0.5^{\circ}$ steps with $1 \mathrm{~s}$ acquisition time per frame.

We used the software DIOPTAS to integrate and calibrate the powder diffraction patterns. ${ }^{8}$ After the integration of the diffraction patterns the structure was refined by Rietveld refinement using the software package GSASII. ${ }^{910}$ For the single crystal diffraction the diffractometer/detector geometry was calibrated by measuring an enstatite single crystal. Afterwards the reflections were indexed and integrated employing CrysAlis ${ }^{\mathrm{PRO}} 39.46 .{ }^{11}$. The structure solution was performed with SHELXT and the refinement with the software package JANA2006. ${ }^{12} 13$

\section{Density functional theory-based calculations}

First-principles calculations were carried out within the framework of density functional theory (DFT), employing the Perdew-Burke-Ernzerhof (PBE) exchange-correlation functional and the plane wave/pseudopotential approach implemented in the CASTEP simulation package. ${ }^{14 \cdots 16}$ "On the fly" norm-conserving or ultrasoft pseudopotentials generated using the descriptors in the CASTEP data base were employed in conjunction with plane waves up to a kinetic energy cutoff of $1020 \mathrm{eV}$ or $630 \mathrm{eV}$, for norm-conserving and ultrasoft pseudopotentials, respectively. The accuracy of the pseudopotentials is well established. ${ }^{17}$ A Monkhorst-Pack grid was used for Brillouin zone integrations. ${ }^{18}$. We used a distance between grid points of $<0.023 \AA^{-1}$. Convergence criteria for geometry optimization included an energy change of $<5 \times 10^{-6} \mathrm{eV}$ atom $^{-1}$ between steps, a maximal force of $<0.008 \mathrm{eV} / \AA$, and a maximal component of the stress tensor $<0.02 \mathrm{GPa}$. Phonon frequencies were obtained from density functional perturbation theory (DFPT) calculations. Raman intensities were computed using DFPT with the " $2 n+1$ " theorem approach. ${ }^{19}$

\section{Synchrotron X-ray diffraction}

We used high-pressure synchrotron powder diffraction to analyzed the powder diffraction data recorded for $\mathrm{Sr}_{3}\left[\mathrm{CO}_{4}\right] \mathrm{O}$ at elevated pressures. At 20(1) GPa the Rietveld refinement (Fig. S 2) was carried out in the tetragonal space group $I 4 / \mathrm{mcm}$ (No. 140) with $Z=4$. The anisotropic displacement parameter of the symmetrically independent strontium as well as of the oxygen atoms were constrained to be identical and we applied restraints to ensure that the $\mathrm{C}-\mathrm{O}$ bond distances are $\approx 1.4 \AA$ and the $\mathrm{O}-\mathrm{C}-\mathrm{O}$ angles are $\approx 109.5^{\circ}$. The high background is mainly due to Compton scattering.

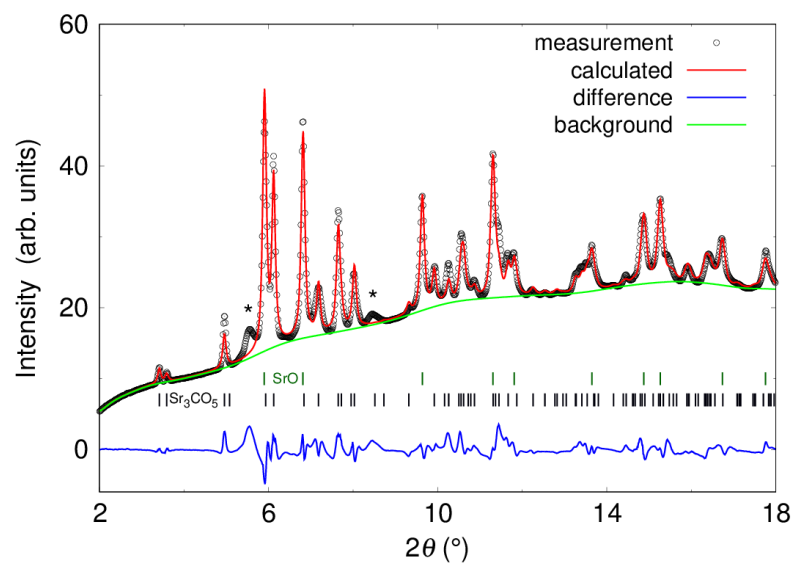

Figure S 2: Rietveld refinement of $\mathrm{Sr}_{3}\left[\mathrm{CO}_{4}\right] \mathrm{O}$ obtained from the high-pressure and temperature synthesis at 20(1) GPa in a DAC. The refinement was carried out in SG I4/ $\mathrm{mcm}$ (No. 140) using $\lambda=0.2906 \AA$. Reflection positions are indicated by tick marks and the residuals between measurement and refinement are shown by the blue line. $\mathrm{SrO}$ is induced as a minor phase and $\mathrm{Sr}_{2}\left[\mathrm{CO}_{4}\right]$ reflections are marked by an asterisk $(*)$.

Table $\mathrm{S} 1$ shows the crystallographic parameters of tetragonal $\mathrm{Sr}_{3}\left[\mathrm{CO}_{4}\right] \mathrm{O}$ at 20(1) GPa obtained by Rietveld refinement in comparison to DFT-based calculations. At ambient conditions we used synchrotron single crystal diffraction to obtain a suitable data set for the structure solution. Table $S 1$ also shows the crystallographic parameters of orthorhombic $\mathrm{Sr}_{3}\left[\mathrm{CO}_{4}\right] \mathrm{O}(P n m a$, No. 62) at ambient conditions from the structure solution and DFT calculations.

Table. S 1: Structural parameters of $\mathrm{Sr}_{3}\left[\mathrm{CO}_{4}\right] \mathrm{O}$ at 20(1) GPa from Rietveld refinement and at ambient conditions from single crystal structure solution in comparison to DFT calculations.

\begin{tabular}{|c|c|c|c|c|}
\hline & exp. & DFT & exp. & DFT \\
\hline pressure & \multicolumn{2}{|c|}{$20 \mathrm{GPa}$} & \multicolumn{2}{|c|}{ ambient conditions } \\
\hline $\begin{array}{l}\text { environment } \\
\text { sample }\end{array}$ & $\begin{array}{l}\text { DAC } \\
\text { powd }\end{array}$ & & $\begin{array}{l}\text { gask } \\
\text { SC }\end{array}$ & \\
\hline
\end{tabular}

\section{Crystal data}

crystal system

space group

$a(\AA)$

$b(\AA)$

$c(\AA)$

$V\left(\AA^{3}\right)$

$Z$

$\rho\left(\mathrm{g} \mathrm{cm}^{-3}\right)$

Refinement

\section{$R_{\text {int }}$}

$w R(\%)$

No. of reflections

No. of parameters

No. of constraints

No. of restraints

\begin{tabular}{llll}
\multicolumn{2}{c}{ tetragonal } & \multicolumn{2}{r}{ orthorhombic } \\
\multicolumn{2}{c}{ I4/ $\mathrm{mcm}$} & \multicolumn{2}{c}{ Pnma } \\
$6.562(1)$ & 6.4904 & $9.892(1)$ & 9.9919 \\
$6.562(1)$ & 6.4904 & $7.131(2)$ & 7.1491 \\
$9.751(3)$ & 9.7150 & $6.806(1)$ & 6.8422 \\
$419.9(1)$ & 409.25 & $480.1(2)$ & 488.76 \\
4 & 4 & 4 & 4 \\
5.61 & 5.76 & 4.91 & 4.82
\end{tabular}

$-\quad-6.4$

$3.9 \quad-\quad 6.5$

$82 \quad-\quad 987$

$20 \quad-\quad 33$

2

10 
Table $\mathrm{S} 2$ shows the atomic coordinates and isotropic displacement parameters of $\mathrm{Sr}_{3}\left[\mathrm{CO}_{4}\right] \mathrm{O}$ at 20(1) $\mathrm{GPa}$ (tetragonal) from Rietveld refinement in comparison to the results obtained by DFT-based calculations. In addition, Table S 2 lists the atomic coordinates and isotropic displacement parameters of $\mathrm{Sr}_{3}\left[\mathrm{CO}_{4}\right] \mathrm{O}$ at ambient conditions (orthorhombic) from the single crystal structure solution and the DFT-based calculations.

Table. S 2: Atomic coordinates and isotropic displacement parameters of $\mathrm{Sr}_{3}\left[\mathrm{CO}_{4}\right] \mathrm{O}$ at 20(1) GPa from Rietveld refinement and single crystal structure solution at ambient conditions $\left(1^{\text {st }}\right.$ line) and DFT-calculations ( $2^{\text {nd }}$ line). For the Rietveld refinement at ambient conditions the $U_{\text {iso }}$ of the symmetrically independent strontium and oxygen atoms were constrained to be identical.

\begin{tabular}{|c|c|c|c|c|c|}
\hline Atom & Site & $x$ & $y$ & $z$ & $U_{\text {iso }}\left(\AA^{2}\right)$ \\
\hline \multicolumn{6}{|c|}{ tetragonal $(I 4 / \mathrm{mcm}), 20 \mathrm{GPa}$} \\
\hline \multirow[t]{2}{*}{ Sr1 } & $8 h$ & $0.187(1)$ & $0.687(1)$ & 0.0 & $0.003(2)$ \\
\hline & & 0.178 & 0.678 & 0.0 & - \\
\hline \multirow[t]{2}{*}{ Sr2 } & $4 a$ & 0.0 & 0.0 & $1 / 4$ & $0.003(2)$ \\
\hline & & 0.0 & 0.0 & $1 / 4$ & - \\
\hline \multirow[t]{2}{*}{$\mathrm{O} 1$} & $16 l$ & $0.125(1)$ & $0.625(1)$ & $0.666(1)$ & $0.02(1)$ \\
\hline & & 0.123 & 0.623 & 0.666 & - \\
\hline \multirow[t]{2}{*}{$\mathrm{O} 2$} & $4 c$ & 0.0 & 0.0 & 0.0 & $0.02(1)$ \\
\hline & & 0.0 & 0.0 & 0.0 & - \\
\hline \multirow[t]{2}{*}{$\mathrm{C}$} & $4 b$ & 0.0 & $1 / 2$ & $1 / 4$ & $0.07(3)$ \\
\hline & & 0.0 & $1 / 2$ & $1 / 4$ & - \\
\hline \multicolumn{6}{|c|}{ orthorhombic (Pnma), ambient conditions } \\
\hline \multirow[t]{2}{*}{ Sr1 } & $4 d$ & $0.4916(1)$ & $3 / 4$ & $0.3574(1)$ & $0.015(1)$ \\
\hline & & 0.4929 & $3 / 4$ & 0.3585 & - \\
\hline \multirow[t]{2}{*}{ Sr2 } & $8 d$ & $0.8038(1)$ & $0.9963(1)$ & $0.5137(1)$ & $0.017(1)$ \\
\hline & & 0.8045 & 0.9958 & 0.5130 & - \\
\hline \multirow[t]{2}{*}{$\mathrm{O} 1$} & $4 c$ & $0.4984(5)$ & $1 / 4$ & $0.0295(9)$ & $0.013(1)$ \\
\hline & & 0.4952 & $1 / 4$ & 0.0279 & - \\
\hline \multirow[t]{2}{*}{$\mathrm{O} 2$} & $4 c$ & $0.7361(5)$ & $3 / 4$ & $0.2683(3)$ & $0.011(1)$ \\
\hline & & 0.7356 & $3 / 4$ & 0.2696 & - \\
\hline \multirow[t]{2}{*}{$\mathrm{O} 3$} & $4 c$ & $0.4325(4)$ & $0.411(1)$ & $0.3061(7)$ & $0.018(1)$ \\
\hline & & 0.4319 & 0.4113 & 0.3057 & - \\
\hline \multirow[t]{2}{*}{$\mathrm{O} 4$} & $8 d$ & $0.6361(6)$ & $1 / 4$ & $0.300(1)$ & $0.017(1)$ \\
\hline & & 0.6333 & $1 / 4$ & 0.3004 & - \\
\hline \multirow[t]{2}{*}{ C } & $4 c$ & $0.4977(7)$ & $1 / 4$ & $0.235(1)$ & $0.012(1)$ \\
\hline & & 0.4966 & $1 / 4$ & 0.2364 & - \\
\hline
\end{tabular}

\section{Determination of the Bulk modulus}

We determined the bulk modulus of $\mathrm{Sr}_{3}\left[\mathrm{CO}_{4}\right] \mathrm{O}$ and $\mathrm{Sr}_{2}\left[\mathrm{CO}_{4}\right]$ from the $\mathrm{X}$-ray powder diffraction data obtained between $30 \mathrm{GPa}$ and ambient conditions measured during the decompression. We fitted a $2^{\text {nd }}$-order and $3^{\text {rd }}$-order Birch-Murnaghan equation (EOS) to unit cell volume using the software EOSFit7-GUI obtained by Rietveld refinement of the X-ray powder diffraction data (Fig $\leqslant 3$ ). ${ }^{20 \cdots 22}$

We fitted a $2^{\text {nd }}$-order $(K=127(3) \mathrm{GPa})$ and $3^{\text {rd }}$-order $\left(K=132(6) \mathrm{GPa}, K^{\prime}=3.2(8)\right.$ ) Birch-Murnaghan equation of state to unit cell volume between $30 \mathrm{GPa}$ and ambient conditions for $\mathrm{Sr}_{2}\left[\mathrm{CO}_{4}\right]$ and obtained a very similar bulk modulus within the experimental error limits. Due to the phase transition for the tetragonal high-pressure phase

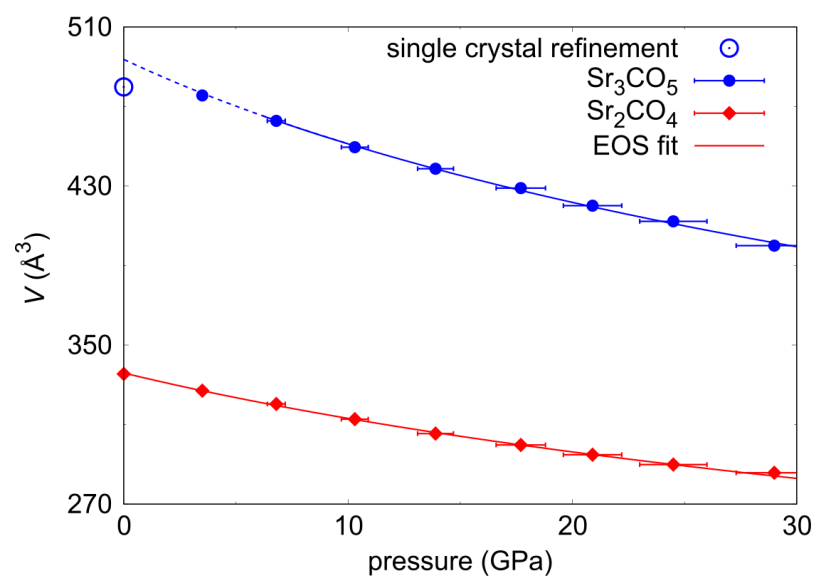

Figure S 3: Bulk modulus of $\mathrm{Sr}_{3}\left[\mathrm{CO}_{4}\right] \mathrm{O}$ and $\mathrm{Sr}_{2}\left[\mathrm{CO}_{4}\right]$ between $30 \mathrm{GPa}$ and ambient conditions.

of $\mathrm{Sr}_{3}\left[\mathrm{CO}_{4}\right] \mathrm{O}$ only the pressure points between $\approx 7 \mathrm{GPa}$ and $30 \mathrm{GPa}$ were considered. We obtained $K=94(7) \mathrm{GPa}$ $\left(2^{\text {nd }}\right.$-order) and $K=103(7) \mathrm{GPa}, K^{\prime}=3.3(8)$ ( $3^{\text {rd }}$-order). Fig. S. 3 also shows the unit cell volume of $\mathrm{Sr}_{3}\left[\mathrm{CO}_{4}\right] \mathrm{O}$ at ambient conditions obtained by single crystal structure solution.

\section{Raman spectroscopy}

Table $\mathrm{S} 3$ lists the peak positions of selected Raman modes of $\mathrm{Sr}_{3}\left[\mathrm{CO}_{4}\right] \mathrm{O}$ and $\mathrm{Sr}_{2}\left[\mathrm{CO}_{4}\right]$ at 20(1) GPa and at ambient conditions obtained by measurements and by DFT-based calculations.

Table. S 3: Peak positions of selected Raman modes of $\mathrm{Sr}_{3}\left[\mathrm{CO}_{4}\right] \mathrm{O}$ and $\mathrm{Sr}_{2}\left[\mathrm{CO}_{4}\right]$ at 20(1) GPa and at ambient conditions from measurements and DFT-based calculations.

\begin{tabular}{cccc|cccc}
\hline \hline \multicolumn{3}{c|}{$\mathrm{Sr}_{3}\left[\mathrm{CO}_{4}\right] \mathbf{O}$} & \multicolumn{4}{c}{$\mathbf{S r}_{2}\left[\mathrm{CO}_{4}\right]$} \\
exp. & DFT & exp. & DFT & exp. & DFT & exp. & DFT \\
\hline \multicolumn{2}{c|}{ 20 GPa } & \multirow{2}{*}{$\mathbf{0 ~ G P a}$} & \multicolumn{3}{|c}{ 20 GPa } & 0 GPa \\
& & & 151 & 141 & & \\
& & & 169 & 159 & & \\
& & & 236 & 219 & & \\
156 & 150 & & & 284 & $271 / 278$ & & \\
189 & 183 & & & 302 & 289 & & \\
206 & 195 & & & 335 & $311 / 317$ & 496 & 471 \\
270 & 269 & & & 538 & 513 & 516 & 489 \\
343 & 337 & & & 561 & 536 & 524 & 494 \\
533 & 504 & 506 & 479 & 576 & 552 & 640 & 605 \\
589 & 560 & 519 & 495 & 701 & 675 & 648 & 617 \\
704 & 677 & 660 & 631 & 732 & 706 & 676 & 645 \\
760 & 730 & 676 & $642 / 645$ & 745 & 721 & 689 & 664 \\
952 & 925 & 864 & 838 & 961 & 937 & 884 & 856 \\
\hline \hline
\end{tabular}




\section{References}

(1) Nguyen Thanh, T.; Bosak, A.; Bauer, J. D.; Luchitskaia, R.; Refson, K.; Milman, V.; Winkler, B. Lattice dynamics and elasticity of $\mathrm{SrCO}_{3}$. J. Appl. Cryst. 2016, 49, 1-9, DOI: 10.1107/S1600576716014205

(2) Boehler, R. New diamond cell for single-crystal Xray diffraction. Rev. Sci. Instrum. 2006, 77, 115103-1115103-3, DOI: 10.1029/JB091iB05p04673

(3) Mao, H. K.; Xu, J.; Bell, P. M. Calibration of the ruby pressure gauge to $800 \mathrm{kbar}$ under quasi-hydrostatic conditions. J. Geophys. Res. 1986, 91, 4673-4676, DOI: 10.1029/JB091iB05p04673

(4) Bayarjargal, L.; Fruhner, C.-J.; Schrodt, N.; Winkler, B. $\mathrm{CaCO}_{3}$ phase diagram studied with Raman spectroscopy at pressures up to $50 \mathrm{GPa}$ and high temperatures and DFT modeling. Phys. Earth Planet. Inter. 2018, 281, 31-45, DOI: 10.1016/j.pepi.2018.05.002

(5) Wojdyr, M. Fityk: a general-purpose peak fitting program. J. Appl. Cryst. 2010, 43, 1126-1128, DOI: $10.1107 /$ S0021889810030499

(6) Benedetti, L. R.; Loubeyre, P. Temperature gradients, wavelength-dependent emissivity, and accuracy of high and very-high temperatures measured in the laser-heated diamond cell. High Press. Res. 2004, 24, 423-455, DOI: 10.1080/08957950412331331718

(7) Liermann, H.-P.; Konôpková, Z.; Morgenroth, W.; Glazyrin, K.; Bednarčik, J.; McBride, E. E.; Petitgirard, S.; Delitz, J. T.; Wendt, M.; Bican, Y.; Ehnes, A.; Schwark, I.; Rothkirch, A.; Tischer, M.; Heuer, J.; Schulte-Schrepping, H.; Kracht, T.; Franz, $\mathrm{H}$. The Extreme Conditions Beamline P02.2 and the Extreme Conditions Science Infrastructure at PETRAIII. J. Synchrotron Radiat. 2014, 22, 908-924, DOI: 10.1107/S1600577515005937

(8) Prescher, C.; Prakapenka, V. B. DIOPTAS: a program for reduction of two-dimensional X-ray diffraction data and data exploration. High. Press. Res. 2015, 35, 223-230, DOI: 10.1080/08957959.2015.1059835

(9) Rietveld, H. M. A profile refinement method for nuclear and magnetic structures. J. Appl. Cryst. 1969, 2, 65-71, DOI: 10.1107/S0021889869006558

(10) Toby, B. H.; Von Dreele, R. B. GSAS-II: the genesis of a modern open-source all purpose crystallography software package. J. Appl. Cryst. 2013, 46, 544-546, DOI: $10.1107 /$ S0021889813003531

(11) Agilent, CrysAlis PRO, Yarnton, England, 2014

(12) Sheldrick, G. M. SHELXT — Integrated space-group and crystal-structure determination. Acta. Cryst. 2015, A71, 3-8, DOI: 10.1107/S2053273314026370

(13) Petricek, V.; Dusek, M.; Palatinus, L. Crystallographic Computing System JANA2006: General features. Z. Kristallogr. 2014, 229, 345-352, DOI: 10.1515/zkri2014-1737
(14) Hohenberg, P.; Kohn, W. Inhomogeneous Electron Gas. Phys. Rev. 1967, 136, B864-B871, DOI: 10.1103/PhysRev.136.B864

(15) Perdew, J. P.; Burke, K.; Ernzerhof, M. Generalized Gradient Approximation Made Simple. Phys. Rev. Lett. 1996, 77, 3865-3868, DOI: 10.1103/PhysRevLett.77.3865

(16) Clark, S. J.; Segall, M. D.; Pickard, C. J.; Hasnip, P. J.; Probert, M. I. J.; Refson, K.; Payne, M. C. First principles methods using CASTEP. Z. Kristallogr. 2005, 220, 567-570, DOI: 10.1524/zkri.220.5.567.65075

(17) Lejaeghere, K.; Bihlmayer, G.; Björkman, T.; Blaha, P.; Blügel, S.; Blum, V.; Caliste, D.; Castelli, I. E.; Clark, S. J.; Dal Corso, A. et al. Reproducibility in density functional theory calculations of solids. Science 2016, 351, aad3000, DOI: 10.1126/science.aad3000

(18) Monkhorst, H. J.; Pack, J. D. Special points for Brillouin-zone integrations. Phys. Rev. B 1976, 13, 5188-5192, DOI: 10.1103/PhysRevB.13.5188

(19) Miwa, K. Prediction of Raman spectra with ultrasoft pseudopotentials. Phys. Rev. B 2011, 84, 094304, DOI: 10.1103/PhysRevB.84.094304

(20) Murnaghan, F. The Compressibility of Media under Extreme Pressures. Proc. Natl. Acad. Sci. 1944, 30, 244-247, DOI: 10.1073/pnas.30.9.244

(21) Birch, F. Finite Elastic Strain of Cubic Crystals. Phys. Rev. 1947, 71, 809-824, DOI: 10.1103/PhysRev.71.809

(22) Gonzalez-Platas, J.; Alvaro, M.; Nestola, F.; Angel, R. EosFit7-GUI: a new graphical user interface for equation of state calculations, analyses and teaching. J. Appl. Cryst. 2016, 49, 1377-1382, DOI: $10.1107 /$ S1600576716008050 